\title{
CELSR1 Promotes Neuroprotection in Cerebral Ischemic Injury Mainly through the Wnt/PKC Signaling Pathway
}

\author{
Li-Hong Wang ${ }^{1,+}$, Geng-Lin Zhang ${ }^{2,+}{ }^{+}$Xing-Yu Liu ${ }^{1}$, Ai Peng ${ }^{1}$, Hai-Yuan Ren ${ }^{1}$, \\ Shu-Hong Huang ${ }^{3}$, Ting Liu ${ }^{1}$ and Xiao-Jing Wang ${ }^{1,4, *}$ \\ 1 Department of Cell Biology, School of Basic Medical Sciences, Shandong University, Jinan 250012, Shandong, \\ China; wanglihong1202@163.com (L.-H.W.); 201714954@mail.sdu.edu.cn (X.-Y.L.); \\ pengai1007@163.com (A.P.); ren15993562826@163.com (H.-Y.R.); liuting@sdu.edu.cn (T.L.) \\ 2 Key Laboratory for Biotech-Drugs Ministry of Health and Key Laboratory for Rare \& Uncommon Diseases of \\ Shandong Province, Shandong Medicinal Biotechnology Center, Shandong First Medical University \& \\ Shandong Academy of Medical Sciences, Jinan 250062, Shandong, China; zgelin@163.com \\ 3 Institute of Basic Medicine, Shandong First Medical University \& Shandong Academy of Medical Sciences, \\ Jinan 250062, Shandong, China; shuhonghuang@sdu.edu.cn \\ 4 Advanced Medical Research Institute, Shandong University, Jinan 250012, Shandong, China \\ * Correspondence: luckingwang@sdu.edu.cn; Tel.: +86-531-8838-2046 \\ + These authors contributed equally to this work.
}

Received: 28 December 2019; Accepted: 10 February 2020; Published: 13 February 2020 updates

\begin{abstract}
Cadherin epidermal growth factor (EGF) laminin G (LAG) seven-pass G-type receptor 1 (CELSR1) is a member of a special subgroup of adhesion G protein-coupled receptors. Although Celsr 1 has been reported to be a sensitive gene for stroke, the effect of CELSR1 in ischemic stroke is still not known. Here, we investigated the effect of CELSR1 on neuroprotection, neurogenesis and angiogenesis in middle cerebral artery occlusion (MCAO) rats. The mRNA expression of Celsr1 was upregulated in the subventricular zone (SVZ), hippocampus and ischemic penumbra after cerebral ischemic injury. Knocking down the expression of Celsr 1 in the SVZ with a lentivirus significantly reduced the proliferation of neuroblasts, the number of CD31-positive cells, motor function and rat survival and increased cell apoptosis and the infarct volume in MCAO rats. In addition, the expression of p-PKC in the SVZ and peri-infarct tissue was downregulated after ischemia/ reperfusion. Meanwhile, in the dentate gyrus of the hippocampus, knocking down the expression of Celsr1 significantly reduced the proliferation of neuroblasts; however, it had no influence on motor function, cell apoptosis or angiogenesis. These data indicate that CELSR1 has a neuroprotective effect on cerebral ischemia injury by reducing cell apoptosis in the peri-infarct cerebral cortex and promoting neurogenesis and angiogenesis, mainly through the Wnt/PKC pathway.
\end{abstract}

Keywords: CELSR1; neurogenesis; angiogenesis; stroke; Wnt/PKC pathway

\section{Introduction}

Stroke is the second-most fatal disease worldwide [1], and approximately $87 \%$ of stroke cases are ischemic stroke triggered by blood flow blockage within major cerebral arteries. Currently, the only FDA-approved treatment for acute ischemic stroke is intravenous recombinant tissue plasminogen activator (tPA) [2], but the majority of patients cannot benefit from this agent due to its narrow treatment time window and association with hemorrhagic complications [3,4]. Therefore, it is necessary to find other more effective therapies for cerebral ischemia. 
Cerebral ischemic can induce spontaneous neurological repair processes, including neurogenesis and angiogenesis [5,6]. Adult neurogenesis occurs mainly in the subventricular zone (SVZ) of the lateral ventricles and the subgranular zone (SGZ) in the hippocampal dentate gyrus (DG) $[7,8]$. In the middle cerebral artery occlusion (MCAO) model, neural stem cells (NSCs) in the SVZ can generate many neuroblasts and migrate to the ischemic penumbra, improving neurological functional recovery [9]. Cerebral ischemia injury can stimulate the expression of endogenous vascular-related factors, thereby promoting the rapid proliferation of vascular endothelial cells, and migrate to the damaged areas to form new blood vessels, providing nutrients and oxygen for the neurons in the ischemic penumbra $[5,10]$.

CELSR1 is an adhesion G protein-coupled receptor [11-14]. Celsr1 was identified as a susceptibility gene for ischemic stroke in Japanese individuals by a genome-wide association study $[15,16]$. Recent evidence has suggested that CELSR1 regulates the direction of dendrite initiation sites $[17,18]$. In vitro, CELSR1 is a positive regulator of endothelial cell migration and angiogenesis [19]. In addition, CELSR1 is also a key component of the noncanonical Wnt/planar cell polarity (PCP) pathway, and it is involved with Fzd3, Fzd6, Dvl1, Dvl2 and Vangl2 in the Wnt/PCP pathway [20,21]. In this decade, more studies have indicated that the noncanonical Wnt/PCP pathway also regulates endothelial cell proliferation and angiogenesis [22-24]. Thus far, the role of CELSR1 in cerebral ischemia is still unclear. To address these questions, we administered lentiviral microinjections to MCAO rats to knock down the expression of Celsr1 to assess the role of CELSR1 in neuroprotection, neurogenesis and angiogenesis in cerebral ischemia in an MCAO model.

\section{Results}

\subsection{The Expression Level of Celsr1 Increased Significantly in the Ischemic SVZ and DG}

To test whether CELSR1 participates in the process of cerebral ischemia, we investigated the mRNA expression of Celsr 1 by quantitative RT-PCR after $2 \mathrm{~h}$ of ischemia/ $22 \mathrm{~h}$ of reperfusion. Compared to that in the sham group (100\%), the mRNA expression of Celsr1 in the MCAO group was significantly increased in the SVZ and DG (SVZ: $271.4 \% \pm 48.69 \%, p=0.0180$; DG: $175.9 \% \pm 26.26 \%, p=0.0446$, Figure $1 \mathrm{~A})$, decreased in the ischemic penumbra $(83.74 \% \pm 3.635 \%, p=0.0110$, Figure $1 \mathrm{~A})$, and showed no obvious change in the ischemic core $(58.81 \% \pm 28.14 \%, p=0.2809$, Figure $1 \mathrm{~A})$ and striatum $(71.53 \%$ $\pm 15.89 \%, p=0.1477$, Figure $1 \mathrm{~A})$. These results suggest that CELSR1 may play a role in cerebral ischemic injury. 
A

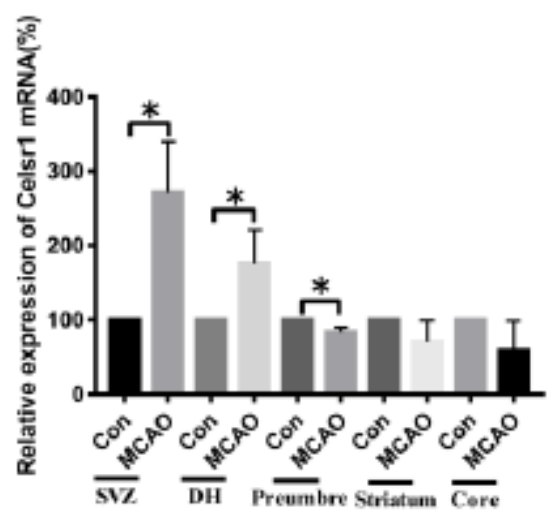

B

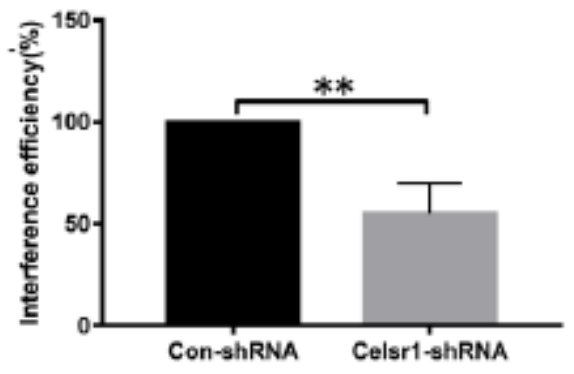

\section{C}

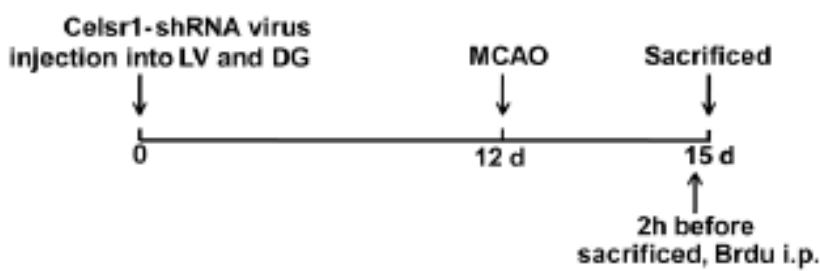

Figure 1. The expression of Celsr1 in the different brain areas of middle cerebral artery occlusion (MCAO) rats. (A) After $2 \mathrm{~h}$ of ischemia/24 h of reperfusion, q-PCR was conducted in the subventricular zone (SVZ), dentate gyrus (DG), ischemic penumbra, ischemic core and ischemic cortex $(n=3$ in each group). ${ }^{*} p<0.05$. (B) The interference efficiency of the Celsr1-shRNA lentivirus in 293 cells was tested by q-PCR ( $n=3$, the experiment was repeated 3 times). ${ }^{* *} p<0.01$. (C) Experimental scheme of pretreatment with the Celsr1-shRNA lentivirus.

\subsection{A Celsr1-shRNA Lentivirus Was Constructed and Microinjected into the Brain}

To identify the roles of CELSR1 in cerebral ischemic injury, a Celsr1-shRNA lentivirus with green fluorescent protein (GFP) was constructed. To test the efficiency of Celsr1 knockdown, HEK293T cells were transfected with the Celsr1-shRNA lentivirus and a control lentivirus. According to the results of quantitative RT-PCR, the interference efficiency of the Celsr1-shRNA lentivirus reached approximately $50 \%$ compared to that of the control lentivirus $(54.88 \% \pm 8.69 \%$ vs. $100 \%, p=0.0016$, Figure $1 \mathrm{~B})$. The Celsr1-shRNA lentivirus and control lentivirus were microinjected into the lateral ventricle and DG of rats. After 12 days, rats underwent MCAO. The rats were sacrificed three days after MCAO (Figure 1C).

\subsection{Knockdown of Celsr1 in the SVZ Accelerated Brain Injury Induced by Ischemia/Reperfusion}

The Celsr1-shRNA lentivirus and control lentivirus were microinjected into the SVZ of rats twelve days before MCAO. After $2 \mathrm{~h}$ of ischemia/70 $\mathrm{h}$ of reperfusion, the rats were sacrificed. Then, the cerebral infarct volume was assessed. The group treated with Celsr1-shRNA lentivirus had a significantly 
larger infarct volume than the control group $(0.06 \% \pm 0.02 \%$ vs. $0.14 \% \pm 0.03 \%, p=0.047$ Figure $2 \mathrm{~A}, \mathrm{~B})$. Neurological deficits were assessed after $22 \mathrm{~h}, 46 \mathrm{~h}$ and of reperfusion following $2 \mathrm{~h}$ of ischemia. The Celsr1-shRNA lentivirus group showed a significantly higher Bederson score than that of the control group at different time points, peaking after $2 \mathrm{~h}$ of ischemia/46 h of reperfusion $(1.26 \pm 0.09 \mathrm{vs}$. $2.2 \pm 0.17, p<0.001$, Figure 2C). Interestingly, we found that most of the rats that died after MCAO were in the Celsr1-shRNA group. Therefore, we calculated the mortality of rats in the control group and Celsr1-shRNA group after MCAO. Compared to that of the control group, the mortality rate caused by ischemia/reperfusion injury in the Celsr1-shRNA lentivirus group increased three-fold (Figure 2D). This shows that Celsr1 knockdown increased the brain infarct volume and neurological deficit score and then led to animal death. This suggests that CELSR1 has a protective effect on cerebral ischemic injury.

A

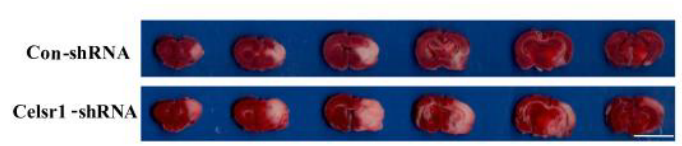

C

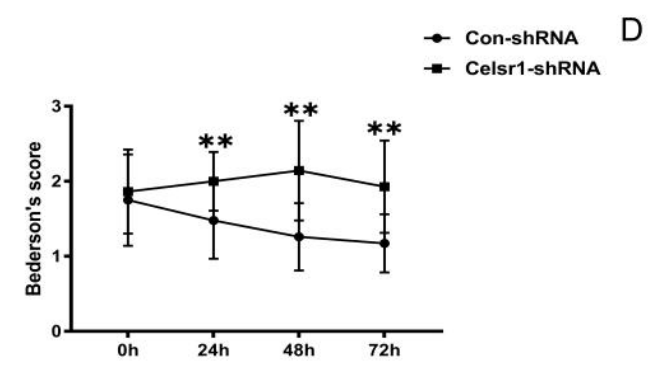

B
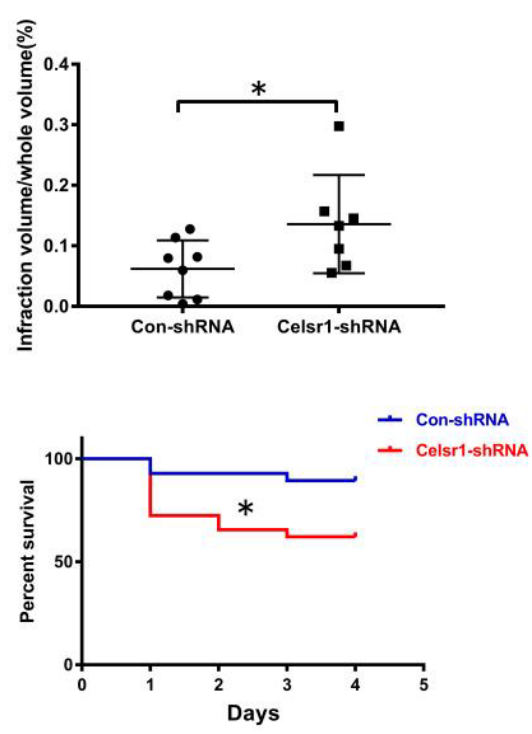

Figure 2. Celsr1 knockdown in the SVZ accelerated brain injury. (A) Triphenyltetrazolium chloride (TTC) staining was used to show the infarct volume in the brain sections. The representative images were placed in order from the anterior to the posterior portion of the brain, from left to right. Scale bar $=10 \mathrm{~mm}$. (B) The infarct volume was quantified by TTC staining. Celsr 1 knockdown significantly increased the infarct volume in the MCAO rats (Con-shRNA group, $n=8$; Celsr1-shRNA group, $n=7$ ). ${ }^{*} p<0.05$. (C) Celsr1 knockdown significantly increased the Bederson score $\left(n=5\right.$ per group, $\left.{ }^{* *} p<0.01\right)$.

(D) The mortality rate was significantly increased in the Celsr1-shRNA group. ${ }^{*} p<0.05$.

\subsection{Celsr1 Knockdown in the SVZ Increased the Percentage of Apoptotic Cells in the Peri-infarct Cerebral Cortex}

The mechanism of the neuroprotective effect of CELSR1 was explored. Can Celsr1 knockdown promote cell apoptosis in the peri-infarct cerebral cortex to increase brain injury? Cleaved caspase- 3 and TdT-mediated dUTP-biotin nick end labeling (TUNEL) staining were applied to brain sections. Compared with that in the control group, the number of caspase-3-positive cells in the peri-infarct cerebral cortex was increased significantly in the SVZ of the Celsr1-shRNA lentivirus group (59.72 \pm 4.14 vs. $35.5 \pm 2.40, p=0.0001$, Figure 3A,B). Both the cleaved caspase-3-positive cells and TUNEL-positive cells were apoptotic cells. There were more TUNEL-positive cells in the Celsr1-shRNA lentivirus group than in the control group $(129.10 \pm 5.34$ vs. $80.58 \pm 9.90, p=0.0001$, Figure $3 C, D)$. These results indicate that Celsr1 knockdown in the SVZ can increase the number of apoptotic cells in the peri-infarct cerebral cortex. 


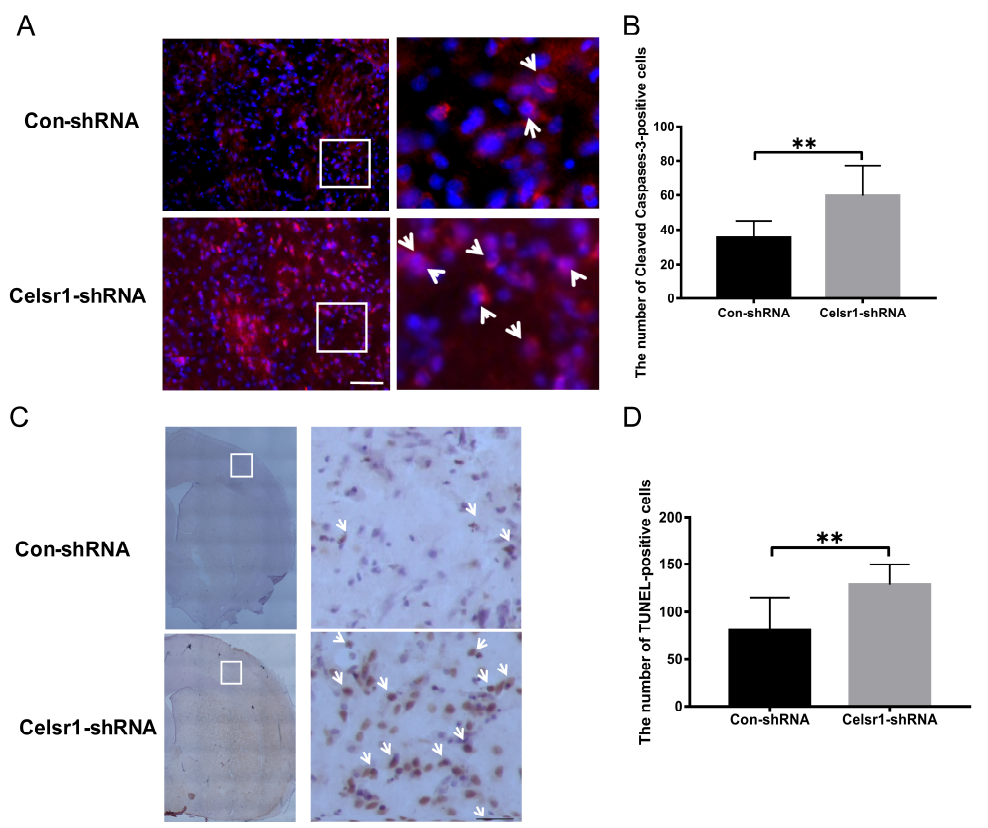

Figure 3. Celsr1 knockdown in the SVZ increased the number of apoptotic cells in the ischemic penumbra. (A) Cleaved caspase-3-positive cells (red) were identified by immunohistochemical staining, and 4,6-diamidino-2-phenylindole (DAPI) (blue) was used to label the nuclei. The insets white box to show photomicrographs of the cells at higher magnification. Arrows point to caspase-3-positive cells. Scale bar $=45 \mu \mathrm{m}$. (B) Quantitative analysis of the number of caspase-3-positive cells in the ischemic penumbra (Con-shRNA group, $n=5$; Celsr1-shRNA group, $n=4$ ). (C) TdT-mediated dUTP-biotin nick end labeling (TUNEL) staining of apoptotic cells in the ischemic penumbra. The insets white box to show photomicrographs of the ischemic penumbra at higher magnification. Arrows point to TUNEL-positive cells. Scale bar $=45 \mu \mathrm{m}$. (D) Quantitative analysis of TUNEL-positive cells in the ischemic penumbra (Con-shRNA group, $n=5$; Celsr1-shRNA group, $n=4$ ). ${ }^{* *} p<0.01$.

\subsection{Celsr1 Knockdown in the SVZ Inhibited Neurogenesis and Angiogenesis after Cerebral Ischemia}

Another mechanism by which CELSR1 exerts neuroprotection was explored by investigating neurogenesis and angiogenesis in the SVZ. BrdU (a marker that labels newborn cells) and DCX (an immature neuronal marker) were used to identify neurogenesis in the SVZ. There were fewer BrdU/nestin-positive cells in the SVZ of the Celsr1-shRNA lentivirus group than in the SVZ of the control group $(68.91 \% \pm 5.41 \%$ vs. $40.00 \% \pm 5.29 \%, p=0.0008$, Figure $4 A, B$ ). CD31 (a marker of vascular endothelial cells) was used to evaluate angiogenesis around the SVZ. The Celsr1-shRNA lentivirus group had a lower CD31-positive vascular area ratio around the SVZ $(12.88 \% \pm 2.11 \%)$ than the control group $(24.61 \% \pm 3.68 \%, p=0.0109$, Figure $4 \mathrm{C}, \mathrm{D})$. These results indicate that Celsr 1 knockdown inhibits neurogenesis and angiogenesis in the SVZ. 
A

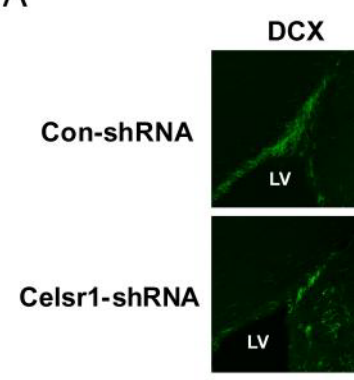

C

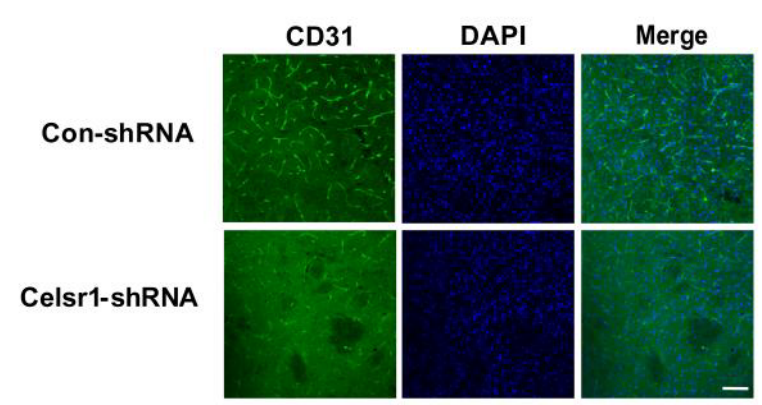

B

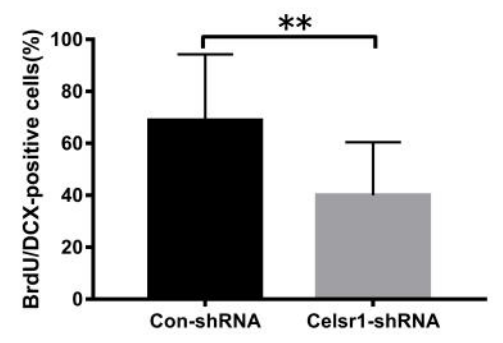

D

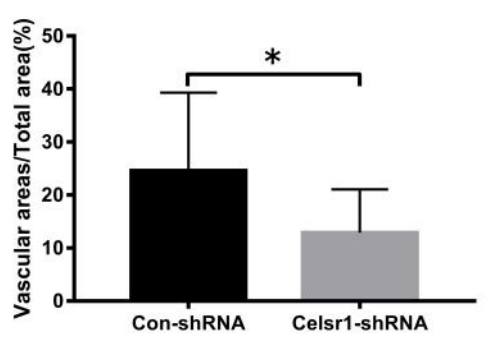

Figure 4. Celsr1 knockdown in the SVZ reduced neurogenesis and angiogenesis. (A) Celsr 1 knockdown in the SVZ significantly reduced the number of BrdU/DCX-positive cells. Scale bar $=45 \mu \mathrm{m}$. (B) Quantitative analysis of BrdU/DCX-positive cells in the SVZ (Con-shRNA group, $n=5$; Celsr1-shRNA group, $n=4$ ). ${ }^{* *} p<0.01$. (C) CD31 staining (green) in the ischemic penumbra. DAPI (blue) was used to label the nuclei. Scale bar $=45 \mu \mathrm{m}$. (D) Quantitative analysis of CD31-positive cells upon Celsr1 knockdown in the SVZ (Con-shRNA group, $n=5$; Celsr1-shRNA group, $n=5$ ). ${ }^{*} p<0.05$.

\subsection{Celsr1 Knockdown in the SVZ Suppressed the Wnt/PKC Signaling Pathway after Cerebral Ischemia}

To deeply clarify the neuroprotective mechanism of CELSR1 in cerebral ischemic injury, tissues of the SVZ and ischemic penumbra from the control group and Celsr1-shRNA group were harvested. The results showed that the expression level of p-PKC was significantly reduced in two brain areas in the Celsr1-shRNA group compared to the control group (SVZ: $0.52 \pm 0.12$ vs. $1.09 \pm 0.11, p=0.004$, Figure $5 \mathrm{~A}, \mathrm{~B}$; ischemic penumbra: $0.62 \pm 0.13$ vs. $1.00 \pm 0.07, p=0.0396$, Figure $5 \mathrm{C}, \mathrm{D})$, but the expression levels of PKC, p-JNK, JNK and $\beta$-catenin were not obviously different in the Celsr1-shRNA group compared to the control group. These results indicate that the neuroprotection exerted by CELSR1 in cerebral ischemic injury may occur through the Wnt/PKC signaling pathway. 
A

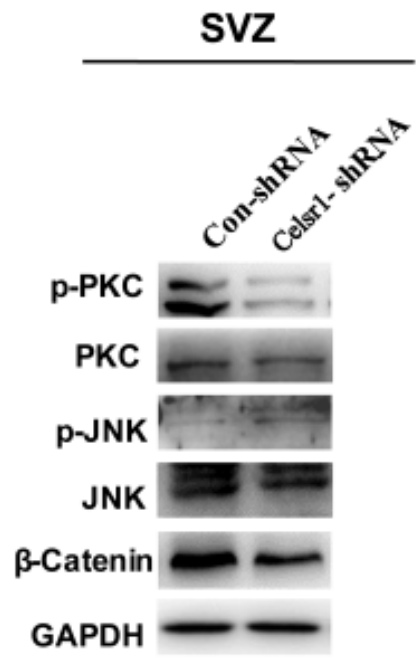

C

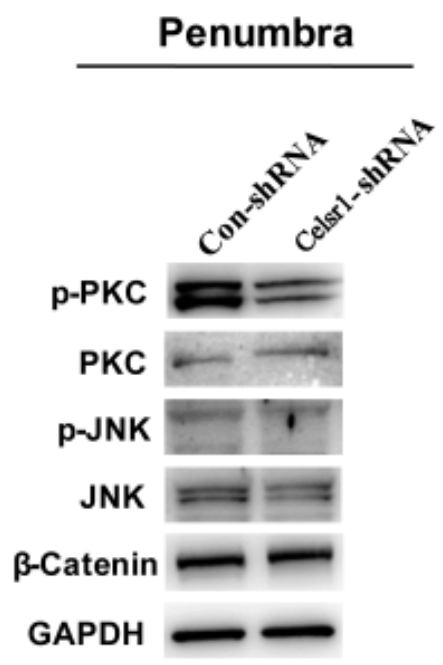

\section{B}

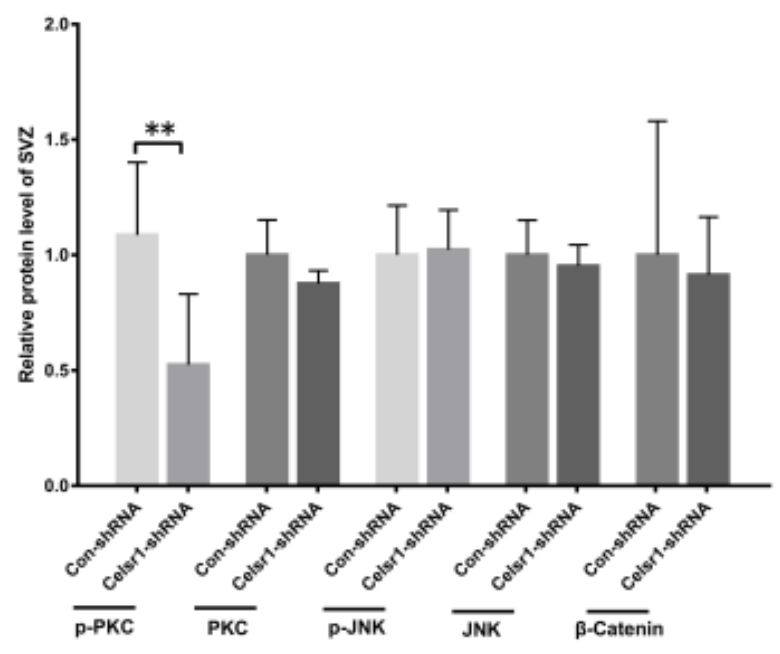

D

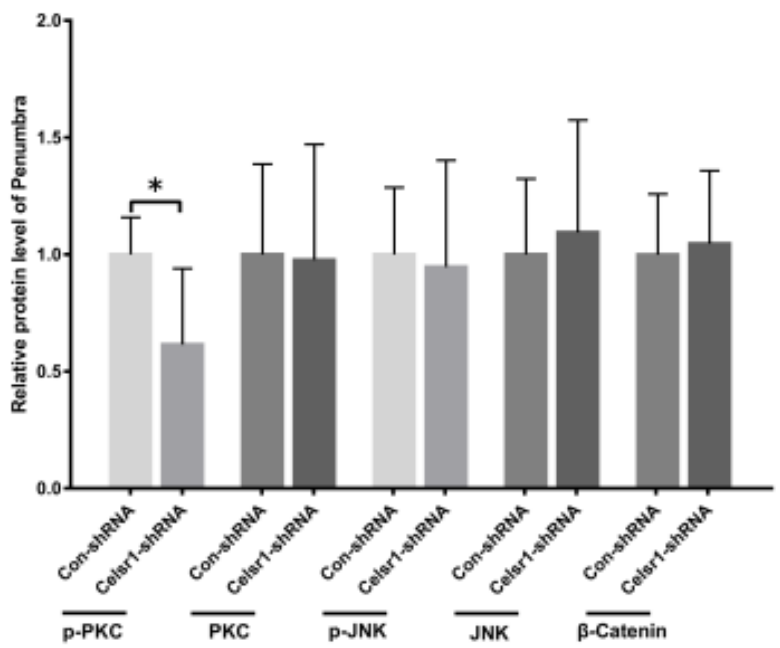

Figure 5. CELSR1 regulates neurogenesis after cerebral ischemia through the Wnt/PKC signaling pathway. (A) Celsr1 knockdown in the SVZ significantly reduced the level of p-PKC, but the levels of $\mathrm{p}$-JNK and $\beta$-catenin did not change. (B) The protein levels in the SVZ were quantified relative to the level of GAPDH. (C) The level of p-PKC was significantly reduced in the ischemic penumbra. (D) The protein levels in the penumbra were relative to the level of GAPDH, and three independent experiments were performed. The reported data represent the mean of the three experiments, Student's $t$-test, ${ }^{*} p<0.05,{ }^{* *} p<0.01$ (Con-shRNA group, $n=6$; Celsr1-shRNA group, $n=6$ ).

2.7. Celsr1 Knockdown in the DG Had no Influence on Cell Apoptosis in the Peri-infarct Cerebral Cortex or Neurological Deficit Scores

To deeply test the neuroprotection exerted by CELSR1 in cerebral ischemic injury, the Celsr1-shRNA lentivirus and control lentivirus were microinjected into the DG of the hippocampus twelve days before MCAO. Immunostaining of cleaved caspase-3 and TUNEL staining were used to identify cell 
apoptosis in the peri-infarct cerebral cortex. The numbers of cleaved caspase-3-positive cells $(34 \pm 2.29$ vs. $41.36 \pm 3.38, p=0.0814$, Figure $6 \mathrm{~A}, \mathrm{~B})$ and TUNEL-positive cells (117.50 \pm 9.20 vs. $110.40 \pm 5.86$, $p=0.5056$, Figure $6 \mathrm{C}, \mathrm{D})$ were not significantly different between the Celsr1-shRNA lentivirus group and the control group. Similarly, we found that the neurological deficit score of the Celsr1-shRNA lentivirus group was not significantly different from that of the control group $(1.67 \pm 0.33$ vs. $1.33 \pm$ $0.33, p=0.5185$, Figure $6 \mathrm{E}$ ). These results indicate that CELSR1 in the DG might not be neuroprotective against cell apoptosis in the peri-infarct cerebral cortex.

A

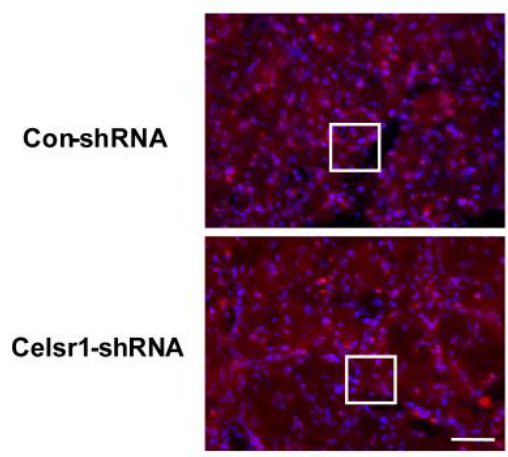

C

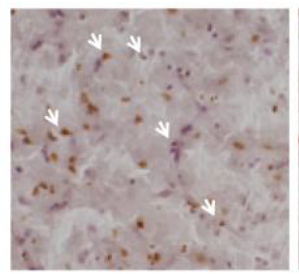

Con-shRNA

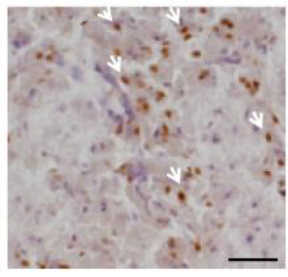

Celsr1-shRNA

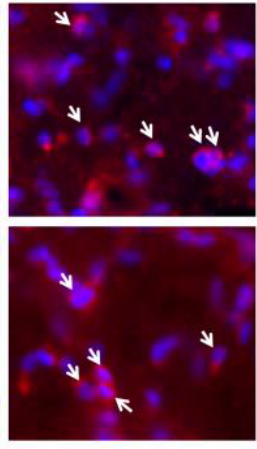

D

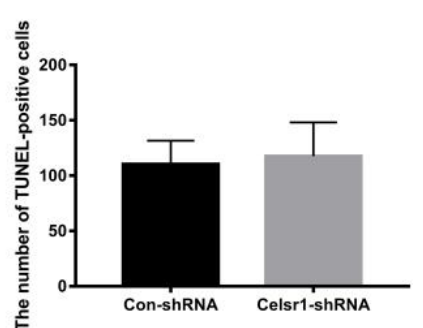

B

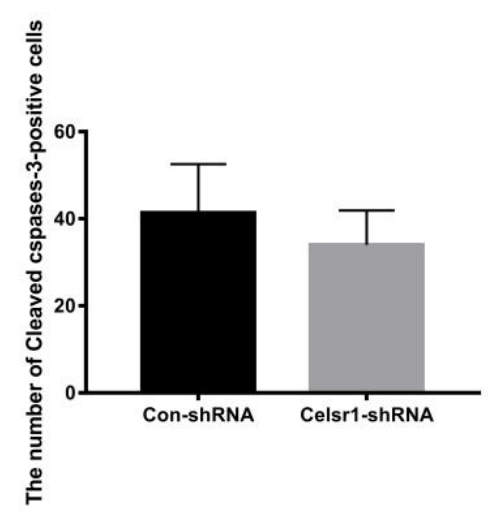

$\mathrm{E}$

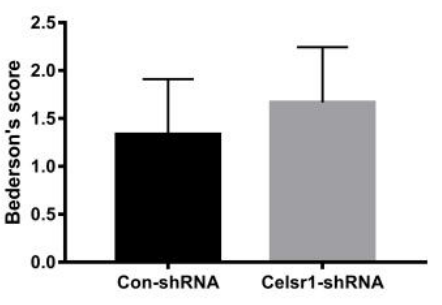

Figure 6. Celsr1 knockdown in the DG had no effect on the number of apoptotic cells in the ischemic penumbra. (A) Cleaved caspase-3 staining (red) in the ischemic penumbra, DAPI (blue) was used to label the nuclei. The insets white box to show photomicrographs of the cells at higher magnification. Arrows point to caspase-3-positive cells. Scale bar $=45 \mu \mathrm{m}$. (B) The number of cleaved caspase-3-positive cells was not different, as determined by quantitative analysis after Celsr1 knockdown in the DG. (C) TUNEL staining was used to show apoptotic cells. Arrows point to TUNEL-positive cells. Scale bar $=45 \mu \mathrm{m}$. (D) No difference was found in the number of TUNEL-positive cells by quantitative analysis ( $n=3$ per group). (E) The Bederson score showed no change after Celsr1 knockdown in the DG ( $n=3$ per group).

\subsection{Celsr1 Knockdown in the DG Inhibited Neurogenesis but did not Affect Angiogenesis after} Cerebral Ischemia

Next, neurogenesis and angiogenesis in the DG were explored. BrdU and DCX were used to identify neurogenesis in the DG. Compared with that in the control group, the number of BrdU/DCX-positive cells in the Celsr1-shRNA group was significantly reduced $(6.00 \% \pm 0.58 \%$ vs. $1.60 \% \pm 0.33 \%$, $p=0.0029$, Figure 7A,B). In the peri-infarct cerebral cortex, the CD31-positive vascular area ratio in the Celsr1-shRNA lentivirus group was similar to that in the control group $(34.91 \% \pm 7.42 \%$ vs. $41.54 \% \pm$ $3.75 \%, p=0.4391$ Figure 7C,D). These results suggest that CELSR1 in the DG promotes neurogenesis but did not affect angiogenesis in the peri-infarct cerebral cortex. 
A
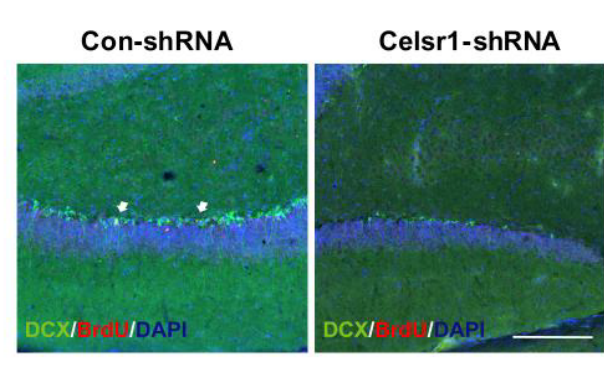

C

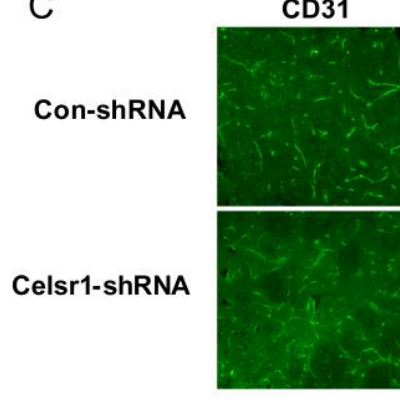

DAPI

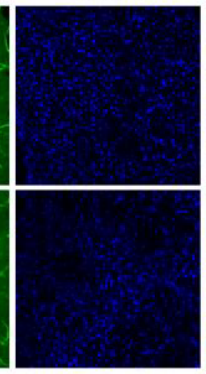

Merge

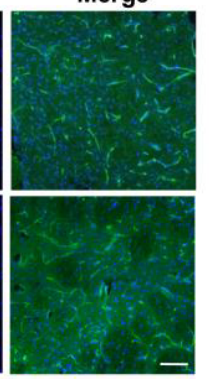

B

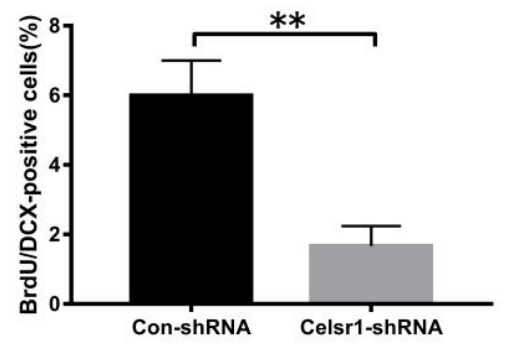

D

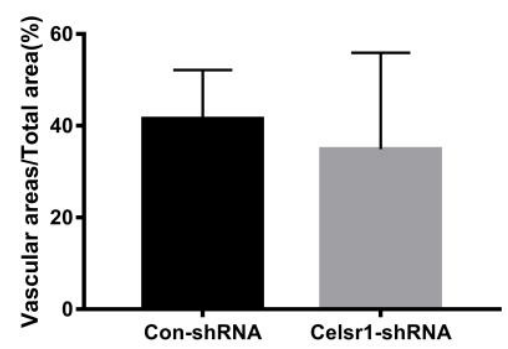

Figure 7. Celsr1 knockdown in the DG promoted neurogenesis but not angiogenesis. (A) The number of BrdU (red)/DCX (green)-positive cells was significantly reduced in the Celsr1-shRNA group. Scale bar $=50 \mu \mathrm{m}$. (B) Quantitative analysis of BrdU/DCX-positive cells in the DG ( $n=3$ per group). ${ }^{* *} p<0.01$.

(C) CD31 staining in the ischemic penumbra upon Celsr1 knockdown in the DG. Scale bar $=45 \mu \mathrm{m}$.

(D) Quantitative analysis of CD31-positive cells in the ischemic penumbra ( $n=3$ per group).

\section{Discussion}

In the present study, we evaluated the neuroprotective effects of CELSR1 on cerebral ischemia in $\mathrm{MCAO}$ rats. The present data provide the first evidence that knocking down the expression of Celsr1 in the SVZ increases cell apoptosis and the infarct volume and reduces neurogenesis and angiogenesis and the motor function and survival rate of MCAO rats. Moreover, the expression of p-PKC was downregulated in the SVZ and peri-infarct tissue. Knocking down Celsr1 expression in the DG only reduced neurogenesis. Therefore, CELSR1 has a neuroprotective effect on cerebral ischemia injury by reducing cell apoptosis in the peri-infarct cerebral cortex and promoting neurogenesis and angiogenesis, mainly through the Wnt/PKC pathway (Figure 8). 

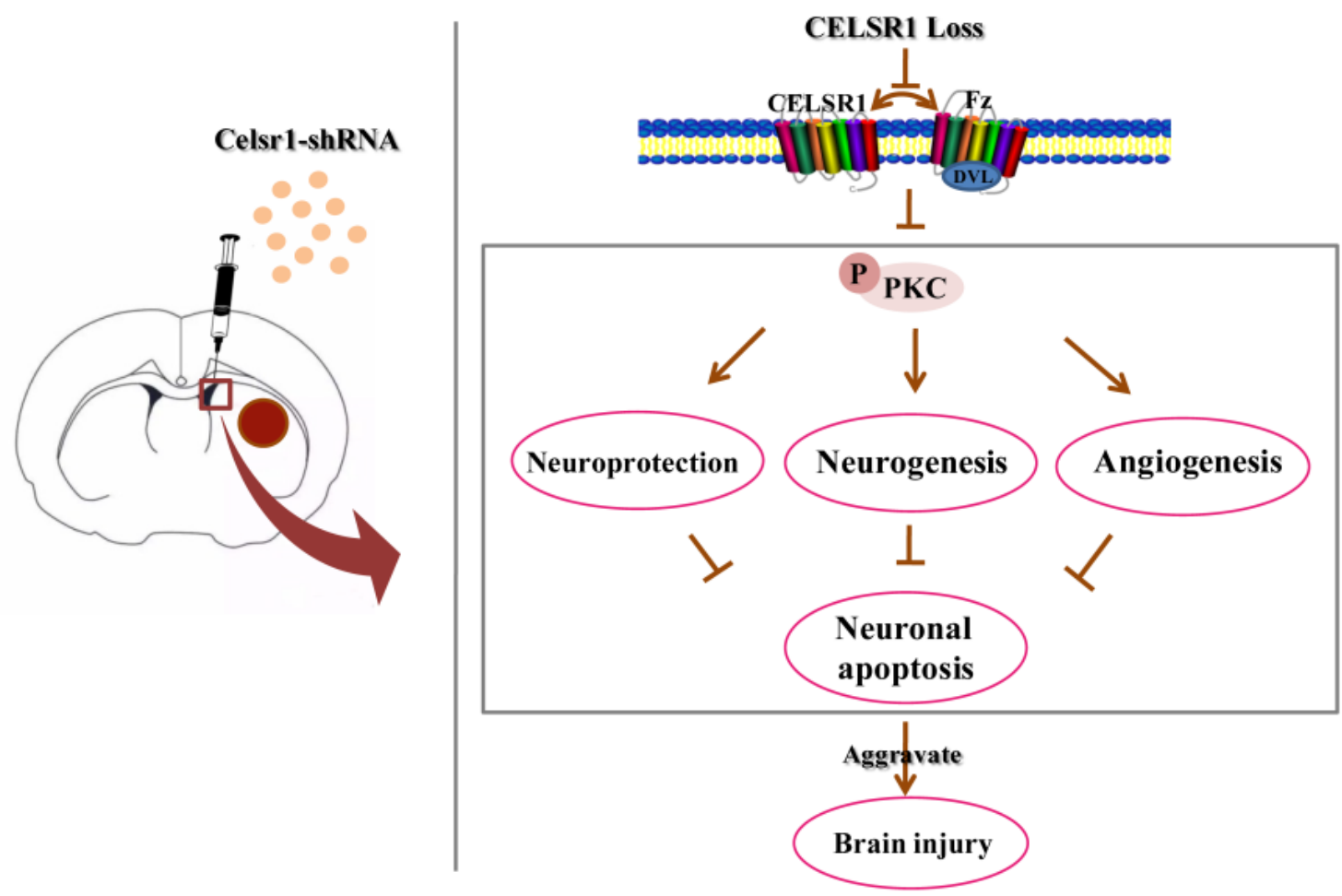

Figure 8. Schematic presentation of our findings showing the role of CELSR1 in the ischemic brain.

In this study, we observed for the first time the neuroprotective effect of CELSR1 on cerebral ischemia injury by knocking down the expression of Celsr1 with a lentivirus in MCAO rats. Although Celsr1 has been identified as a susceptibility gene for ischemic stroke $[15,16]$, the role of CELSR1 in cerebral ischemia injury is unknown. The rats were treated with a Celsr1-shRNA lentivirus twelve days before MCAO was performed. Our data showed that knocking down the expression of Celsr 1 in the SVZ increased the infarct volume, the percentage of apoptotic cells, motor function and the mortality rate. However, knocking down the expression of Celsr1 in the DG did not affect the above indexes. The neuroprotective effect of CELSR1 on cerebral ischemia injury in MCAO rats was found for the first time.

A major observation of this study was that CELSR1 promoted neurogenesis and angiogenesis in MCAO rats. The mRNA expression of Celsr 1 has been reported to be primarily confined to areas of NSCs proliferation, including the ventricular zones during brain development, the telencephalic ependymal zone, and the subgranular layer of dentate gyrus in the adult brain [11]. CELSR1 can regulate the direction of dendrite initiation sites of newborn granule cells during adult hippocampal neurogenesis [17]. Loss of function of Celsr1 in mice results in neural progenitor fate decision defects, cortical hypoplasia and behavioral impairment [18]. CELSR1 plays an important regulatory role in the nervous system. The SVZ of the lateral ventricle and the SGZ of the hippocampal DG are two areas in which neurogenesis occurs in the mammalian adult $[25,26]$. In an MCAO model, ischemic injury can promote neurogenesis in the SVZ and SGZ [27-29]. NSCs in the SVZ can migrate to infarct-damaged areas and become newborn neurons to repair the neural circuit [30-33]. NSCs in the SGZ can migrate to the granular cell layer and become new neurons to reverse the learning and memory dysfunction induced by ischemia [34]. Celsr1 knockdown in the SVZ or DG by shRNA lentivirus inhibited neurogenesis in MCAO rats. In human aortic endothelial cells, CELSR1 promotes cell proliferation and migration, and the formation of capillary-like structures [19]. Angiogenesis in the peri-infarct area is very important for the recovery of cerebral ischemia. Celsr1 knockdown in the SVZ but not the DG inhibited angiogenesis. Celsr1 knockdown in the DG did not affect angiogenesis 
and had no significant neuroprotective effect in MCAO rats. The reason may be that NSCs in the DG are unable to migrate to the cerebral ischemic damage areas near the cortex and striatum.

In addition to canonical Wnt signaling, noncanonical Wnt/ PCP signaling has an important regulatory role in neurogenesis, angiogenesis and cell apoptosis $[17,24]$. The Wnt/PKC, Wnt/JNK and Wnt/ROCK pathways are downstream pathways that are noncanonical [35-37]. CELSR1 is a core protein of the Wnt/PCP pathway. Thus far, the role of CELSR1 in ischemia/reperfusion-induced brain injury has not been reported. To explore the mechanism by which CELSR1 regulates neurogenesis and angiogenesis in MCAO rats, the phosphorylation levels of JNK, PKC and $\beta$-catenin were measured in SVZ and ischemic penumbra. Only the expression level of phosphorylated PKC was significantly reduced when Celsr1 was knocked down. Based on our present study, it seems that CELSR1 regulates neurogenesis and angiogenesis in ischemia/reperfusion injury through the Wnt/PKC pathway, but not the Wnt/JNK pathway or canonical Wnt signaling.

In conclusion, this is the first report to explore the neuroprotective effect of CELSR1 on cerebral ischemia by lentiviral knockdown in MCAO rats. First, CELSR1 in the SVZ was shown to reduce neuronal apoptosis, the infarct volume and the mortality rate and restore motor function. Furthermore, neurogenesis in the SVZ and DG and angiogenesis in the ischemic penumbra were promoted by CELSR1, mainly through the Wnt/PKC pathway. These data demonstrate that CELSR1 plays a critical role in cerebral ischemia/reperfusion injury, which provides a potential target for the clinical treatment of cerebral ischemia. In this study, we verified that loss of CELSR1 protein can aggravate brain injury in rat MCAO models, but due to the limitations of lentivirus, we did not positively verify the role of CELSR1 protein (overexpression of Celsr1) in cerebral ischemic injury. Moreover, the study of the downstream pathways affected by CELSR1 protein is too simple, and there is no detailed study of a series of protein changes downstream of PKC. Our next work will focus on these two aspects.

\section{Materials and Methods}

\subsection{Experimental Model}

Healthy 2-month-old adult male Sprague-Dawley rats weighing 270-300 g were obtained from Vital River Laboratories (Beijing, China) and used for this study. The temperature of the feeding environment was controlled at $22{ }^{\circ} \mathrm{C} \pm 2{ }^{\circ} \mathrm{C}$, and the rats were kept on a 12-h light/dark cycle. All procedures were approved by the Ethics Committee on Animal Experiments of Medical School of Shandong University (No. LL-201702002, 20 May 2017).

\subsection{Intracerebral Microinjection}

After anesthesia with $10 \%$ chloral hydrate, each rat was placed into a stereotaxic frame. The head was further stabilized in a customized head mold. The right lateral ventricle was targeted at the following coordinates from bregma: $-0.9 \mathrm{~mm}$ anterior, $\pm 1.5 \mathrm{~mm}$ lateral and $-3.6 \mathrm{~mm}$ deep. The DG of the hippocampus was targeted at the following coordinates from bregma: $-3.72 \mathrm{~mm}$ anterior, $\pm 2.2 \mathrm{~mm}$ lateral and $-3.4 \mathrm{~mm}$ deep. The Celsr1-shRNA lentivirus and control lentivirus used in this study, with titers ranging from $2 \times 10^{8}$ to $8 \times 10^{8} \mathrm{CFU} / \mathrm{mL}$, were purchased from Shanghai GeneChem Company (Shanghai, China). Two microliters of lentivirus was injected at a rate of $1 \mu \mathrm{L} / \mathrm{min}$, and the needle was retained in place for $5 \mathrm{~min}$ following the injection. Twelve days after injection, the animals underwent MCAO for $2 \mathrm{~h}$ followed by reperfusion for $22 \mathrm{~h}$.

\subsection{Middle Cerebral Artery (MCA) Occlusion Model}

Rats were subjected to MCAO based on a published protocol [38]. Briefly, rats were anesthetized with 10\% chloral hydrate. The right common carotid artery (CCA), external carotid artery (ECA), and internal carotid artery (ICA) were isolated. A nylon filament $(0.28 \mathrm{~mm}$ in diameter) with an expanded tip was gently advanced from the CCA into the lumen of the ICA. The tip of the filament was positioned at the origin of the middle cerebral artery (MCA). The right MCA was occluded with 
the filament for $2 \mathrm{~h}$, and then the filament was withdrawn to allow $22 \mathrm{~h}$ of reperfusion. During recovery from the anesthesia, the animals were returned to their home cages.

\subsection{Examination of Neurological Deficits}

Behavioral assays were performed as previously described [1,38]. Scales from zero to four were used to assess the effects of MCAO on neurological behavior. After 22-h, 46-h or 70-h reperfusion, the rats were scored according to the guidelines.

\subsection{Evaluation of Infarct Volume}

Three days after MCAO, the infarct area was measured by 2\% TTC (Sigma Chemical Co., St. Louis, MO, USA) staining as previously described [1]. The infarct area of each slice was measured with NIH's ImageJ software, version 1.46 (Bethesda, MA, USA), as previously described.

\subsection{BrdU Labeling}

After MCAO $72 \mathrm{~h}$, the rats were injected intraperitoneally with $50 \mathrm{mg} / \mathrm{kg}$ BrdU (Sigma). The rats were perfused $2 \mathrm{~h}$ after the injection (SVZ: Con-shRNA group, $\mathrm{n}=5$; Celsr1-shRNA group, $\mathrm{n}=4$; DG: $\mathrm{n}=3$ in each group). Rats were transcardially perfused with normal saline followed by $4 \%$ paraformaldehyde. The brains were removed and fixed in $4 \%$ paraformaldehyde.

\subsection{Immunohistochemistry}

Serial sagittal or coronal $(40 \mu \mathrm{m})$ sections were cut with a cryostat and stored at $-80{ }^{\circ} \mathrm{C}$. For immunofluorescence staining, the sections were incubated overnight with primary antibody at $4{ }^{\circ} \mathrm{C}$. The primary antibodies used were mouse monoclonal anti-CD31 (1:50; Proteintech Group, Chicago, IL, USA), sheep monoclonal anti-BrdU (1:500; Cell Signaling Technology, Danvers, MA, USA) and rabbit monoclonal anti-cleaved caspase-3 (1:500; Cell Signaling Technology, Danvers, MA, USA). The secondary antibodies used were Alexa Fluor 488-conjugated IgG (1:1000; Invitrogen, Carlsbad, CA, USA) and Alexa Fluor 594-conjugated IgG (1:1000; Invitrogen, Carlsbad, CA, USA). The nuclei of the cells were counterstained with 4,6-diamidino-2-phenylindole (DAPI).

\subsection{Western Blot Analysis}

Three days after MCAO in rats, tissues from the SVZ and ischemic penumbra were harvested. The tissues were lysed in lysis buffer containing $1 \%$ protease inhibitor and $1 \%$ phosphatase inhibitor followed by centrifugation for $15 \mathrm{~min}$ at $14,000 \mathrm{rpm}$. The total protein was separated by SDS-PAGE and transferred to a nitrocellulose membrane. The membrane was blocked and incubated with an appropriate primary antibody and secondary antibody. The following antibodies were used: mouse anti-GAPDH (1:5,000, CST, Danvers, MA, USA), rabbit anti-PKC (1:500, Wanleibio, Shen Yang, China), rabbit anti-JNK (1:500, Wanleibio, Shen Yang, China), rabbit anti- $\beta$-catenin (1:10,000, Proteintech Group, Chicago, IL, USA), rabbit anti-p-PKC (1:10,00, CST, Danvers, MA, USA), rabbit anti-p-JNK (1:500, Wanleibio, Shen Yang, China), and horseradish peroxidase (HRP)-conjugated secondary antibodies (1:10,000; Millipore, Billerica, MA, USA).

\subsection{Quantitative RT-PCR}

Tissues from different areas of the brain were harvested from the MCAO group and the sham operation group. TRIzol was used to extract total RNA, and the RNA was reverse transcribed into cDNA. The resulting cDNA was amplified by PCR in the presence of oligonucleotide primer pairs designed to target Celsr 1 cDNA and $\beta$-actin. The primers used for PCR were as follows: $\beta$-actin fo: gag agg gaa atc gtg cgt gac, re: cat acc cag gaa gga agg ct; Celsr 1 fo: gcc agt ttg ctg ttg ctc, re: gac agg ctt gct tcg ttc. The PCR conditions were $10 \mathrm{~min}$ at $95^{\circ} \mathrm{C}, 39$ cycles at $95^{\circ} \mathrm{C}$ for $15 \mathrm{~s}, 60^{\circ} \mathrm{C}$ for $30 \mathrm{~s}$ and $72{ }^{\circ} \mathrm{C}$ for $30 \mathrm{~s}$, followed by incubation at $72{ }^{\circ} \mathrm{C}$ for $10 \mathrm{~min}$ and maintenance at $4{ }^{\circ} \mathrm{C}$. 


\subsection{TUNEL Staining}

Three days after MCAO, brains were serially sliced in coronal sections at a thickness of $40 \mu \mathrm{m}$ with a cryostat. The sections were assessed by TUNEL assay. The kit was purchased from Promega (Madison, WI, USA), and the experiment was conducted according to the manufacturer's protocol.

\subsection{Cell Transfection}

HEK293T cells were seeded into 6-well plates and cultured at $37^{\circ} \mathrm{C}$ and $5 \% \mathrm{CO}_{2}$ for $24 \mathrm{~h}$. Then, the old culture medium was replaced with fresh medium containing $4 \mu \mathrm{L}$ of Celsr1-shRNA lentivirus with GFP. After $48 \mathrm{~h}$ of viral infection, the cells were harvested, and RNA was extracted.

\subsection{Statistical Analysis}

The results are presented as the mean \pm SEM. Statistical analysis of differences was conducted using Student's $t$-tests. A statistically significant difference was set at $p<0.05$.

\section{Conclusions}

CELSR1 has neuroprotective effects in cerebral ischemia/reperfusion injury, and the protective mechanism of CELSR1 seems mainly through the Wnt/PKC pathway. These findings suggest that CELSR1 may be a potential target for the clinical treatment of cerebral ischemia.

Author Contributions: L.-H.W., G.-L.Z. and X.-J.W. participated in the design of the study, collected the data, performed the statistical analyses and drafted the manuscript; X.-Y.L., A.P., H.-Y.R. and T.L. participated in the design of the study and contributed to the collection of the data; S.-H.H. contributed materials and reagents. All authors have read and agreed to the published version of the manuscript.

Funding: Natural Science Foundation of Shandong Province (ZR2017 MH058), Key research and development plan of Shandong Province (2015GSF118020, 2016GSF120015).

Conflicts of Interest: The authors declare no conflicts of interests. The funders had no role in the design of the study; in the collection, analyses, or interpretation of data; in the writing of the manuscript, or in the decision to publish the results.

\section{Abbreviations}

$\begin{array}{ll}\text { CELSR1 } & \text { Cadherin epidermal growth factor (EGF) laminin G (LAG) seven-pass G-type receptor 1 } \\ \text { MCAO } & \text { middle cerebral artery occlusion } \\ \text { SVZ } & \text { subventricular zone } \\ \text { tPA } & \text { tissue plasminogen activator } \\ \text { SGZ } & \text { subgranular zone } \\ \text { NSCs } & \text { neural stem cells } \\ \text { PCP } & \text { planar cell polarity } \\ \text { GFP } & \text { green fluorescent protein } \\ \text { DG } & \text { dentate gyrus } \\ \text { CCA } & \text { common carotid artery } \\ \text { ECA } & \text { external carotid artery } \\ \text { ICA } & \text { internal carotid artery } \\ \text { MCA } & \text { middle cerebral artery } \\ \text { TTC } & \text { triphenyltetrazolium chloride } \\ \text { TUNEL } & \text { TdT-mediated dUTP-biotin nick end labeling }\end{array}$

\section{References}

1. Hui, Z.; Sha, D.J.; Wang, S.L.; Li, C.S.; Qian, J.; Wang, J.Q.; Zhao, Y.; Zhang, J.H.; Cheng, H.Y.; Yang, H.; et al. Panaxatriol saponins promotes angiogenesis and enhances cerebral perfusion after ischemic stroke in rats. BMC Complement. Altern. Med. 2017, 17, 70. [CrossRef] [PubMed] 
2. Levine, S.R.; Khatri, P.; Broderick, J.P.; Grotta, J.C.; Kasner, S.E.; Kim, D.; Meyer, B.C.; Panagos, P.; Romano, J.; Scott, P. Review, historical context, and clarifications of the NINDS rt-PA stroke trials exclusion criteria: Part 1: Rapidly improving stroke symptoms. Stroke 2013, 44, 2500-2505. [CrossRef] [PubMed]

3. Tilley, B.C.; Lyden, P.D.; Brott, T.G.; Lu, M.; Levine, S.R.; Welch, K.M. Total quality improvement method for reduction of delays between emergency department admission and treatment of acute ischemic stroke. The National Institute of Neurological Disorders and Stroke rt-PA Stroke Study Group. Arch. Neurol. 1997, 54, 1466-1474. [CrossRef] [PubMed]

4. Esposito, E.; Hayakawa, K.; Maki, T.; Arai, K.; Lo, E.H. Effects of Postconditioning on Neurogenesis and Angiogenesis during the Recovery Phase after Focal Cerebral Ischemia. Stroke 2015, 46, 2691-2694. [CrossRef]

5. Zhang, Z.G.; Zhang, L.; Jiang, Q.; Zhang, R.; Davies, K.; Powers, C.; Bruggen, N.; Chopp, M. VEGF enhances angiogenesis and promotes blood-brain barrier leakage in the ischemic brain. J. Clin. Investig. 2000, 106, 829-838. [CrossRef]

6. Ruan, L.; Wang, B.; ZhuGe, Q.; Jin, K. Coupling of neurogenesis and angiogenesis after ischemic stroke. Brain Res. 2015, 1623, 166-173. [CrossRef]

7. Woitke, F.; Ceanga, M.; Rudolph, M.; Niv, F.; Witte, O.W.; Redecker, C.; Kunze, A.; Keiner, S. Adult hippocampal neurogenesis poststroke: More new granule cells but aberrant morphology and impaired spatial memory. PLoS ONE 2017, 12, e0183463. [CrossRef]

8. Lu, J.; Manaenko, A.; Hu, Q. Targeting Adult Neurogenesis for Poststroke Therapy. Stem Cells Int. 2017, 2017, 5868632. [CrossRef]

9. Zhang, R.L.; Zhang, Z.G.; Zhang, L.; Chopp, M. Proliferation and differentiation of progenitor cells in the cortex and the subventricular zone in the adult rat after focal cerebral ischemia. Neuroscience 2001, 105, $33-41$. [CrossRef]

10. Vallee, A.; Guillevin, R.; Vallee, J.N. Vasculogenesis and angiogenesis initiation under normoxic conditions through Wnt/beta-catenin pathway in gliomas. Rev. Neurosci. 2018, 29, 71-91. [CrossRef]

11. Boutin, C.; Goffinet, A.M.; Tissir, F. Celsr1-3 cadherins in PCP and brain development. Curr. Top. Dev. Biol. 2012, 101, 161-183. [PubMed]

12. Hadjantonakis, A.K.; Sheward, W.J.; Harmar, A.J.; de Galan, L.; Hoovers, J.M.; Little, P.F. Celsr1, a neural-specific gene encoding an unusual seven-pass transmembrane receptor, maps to mouse chromosome 15 and human chromosome 22qter. Genomics 1997, 45, 97-104. [CrossRef] [PubMed]

13. Duncan, J.S.; Stoller, M.L.; Francl, A.F.; Tissir, F.; Devenport, D.; Deans, M.R. Celsr1 coordinates the planar polarity of vestibular hair cells during inner ear development. Dev. Biol. 2017, 423, 126-137. [CrossRef] [PubMed]

14. Wang, X.J.; Zhang, D.L.; Xu, Z.G.; Ma, M.L.; Wang, W.B.; Li, L.L.; Han, X.L.; Huo, Y.; Yu, X.; Sun, J.P. Understanding cadherin EGF LAG seven-pass G-type receptors. J. Neurochem. 2014, 131, 699-711. [CrossRef]

15. Yamada, Y.; Fuku, N.; Tanaka, M.; Aoyagi, Y.; Sawabe, M.; Metoki, N.; Yoshida, H.; Satoh, K.; Kato, K.; Watanabe, S.; et al. Identification of CELSR1 as a susceptibility gene for ischemic stroke in Japanese individuals by a genome-wide association study. Atherosclerosis 2009, 207, 144-149. [CrossRef]

16. Zhan, Y.H.; Lin, Y.; Tong, S.J.; Ma, Q.L.; Lu, C.X.; Fang, L.; Wei, W.; Cai, B.; Wang, N. The CELSR1 polymorphisms rs6007897 and rs4044210 are associated with ischaemic stroke in Chinese Han population. Ann. Hum. Biol. 2015, 42, 26-30. [CrossRef]

17. Schafer, S.T.; Han, J.; Pena, M.; von Bohlen Und Halbach, O.; Peters, J.; Gage, F.H. The Wnt adaptor protein ATP6AP2 regulates multiple stages of adult hippocampal neurogenesis. J. Neurosci. 2015, 35, 4983-4998. [CrossRef]

18. Boucherie, C.; Boutin, C.; Jossin, Y.; Schakman, O.; Goffinet, A.M.; Ris, L.; Gailly, P.; Tissir, F. Neural progenitor fate decision defects, cortical hypoplasia and behavioral impairment in Celsr1-deficient mice. Mol. Psychiatry 2018, 23, 723-734. [CrossRef]

19. Zhan, Y.H.; Luo, Q.C.; Zhang, X.R.; Xiao, N.A.; Lu, C.X.; Yue, C.; Wang, N.; Ma, Q.L. CELSR1 Is a Positive Regulator of Endothelial Cell Migration and Angiogenesis. Biochemistry 2016, 81, 591-599. [CrossRef] [PubMed]

20. Tissir, F.; Goffinet, A.M. Atypical cadherins Celsr1-3 and planar cell polarity in vertebrates. Prog. Mol. Biol. Transl. Sci. 2013, 116, 193-214. [PubMed]

21. Tissir, F.; De-Backer, O.; Goffinet, A.M.; Lambert de Rouvroit, C. Developmental expression profiles of Celsr (Flamingo) genes in the mouse. Mech. Dev. 2002, 112, 157-160. [CrossRef] 
22. Qu, Z.; Su, F.; Qi, X.; Sun, J.; Wang, H.; Qiao, Z.; Zhao, H.; Zhu, Y. Wnt/beta-catenin signalling pathway mediated aberrant hippocampal neurogenesis in kainic acid-induced epilepsy. Cell Biochem. Funct. 2017, 35, 472-476. [CrossRef] [PubMed]

23. Liu, A.; Chen, S.; Cai, S.; Dong, L.; Liu, L.; Yang, Y.; Guo, F.; Lu, X.; He, H.; Chen, Q.; et al. Wnt5a through noncanonical Wnt/JNK or Wnt/PKC signaling contributes to the differentiation of mesenchymal stem cells into type II alveolar epithelial cells in vitro. PLoS ONE 2014, 9, e90229. [CrossRef] [PubMed]

24. Choi, H.J.; Park, H.; Lee, H.W.; Kwon, Y.G. The Wnt pathway and the roles for its antagonists, DKKS, in angiogenesis. Iubmb Life 2012, 64, 724-731. [CrossRef]

25. Alvarez-Buylla, A.; Lim, D.A. For the long run: Maintaining germinal niches in the adult brain. Neuron 2004, 41, 683-686. [CrossRef]

26. Zhao, C.; Deng, W.; Gage, F.H. Mechanisms and functional implications of adult neurogenesis. Cell 2008, 132, 645-660. [CrossRef]

27. Jin, K.; Minami, M.; Lan, J.Q.; Mao, X.O.; Batteur, S.; Simon, R.P.; Greenberg, D.A. Neurogenesis in dentate subgranular zone and rostral subventricular zone after focal cerebral ischemia in the rat. Proc. Natl. Acad. Sci. USA 2001, 98, 4710-4715. [CrossRef]

28. Kokaia, Z.; Lindvall, O. Neurogenesis after ischaemic brain insults. Curr. Opin. Neurobiol. 2003, 13, $127-132$. [CrossRef]

29. Wang, X.J.; Sun, T.; Kong, L.; Shang, Z.H.; Yang, K.Q.; Zhang, Q.Y.; Jing, F.M.; Dong, L.; Xu, X.F.; Liu, J.X.; et al. Gypenosides pre-treatment protects the brain against cerebral ischemia and increases neural stem cells/progenitors in the subventricular zone. Int. J. Dev. Neurosci. 2014, 33, 49-56. [CrossRef]

30. Sun, L.; Lee, J.; Fine, H.A. Neuronally expressed stem cell factor induces neural stem cell migration to areas of brain injury. J. Clin. Investig. 2004, 113, 1364-1374. [CrossRef]

31. Thored, P.; Arvidsson, A.; Cacci, E.; Ahlenius, H.; Kallur, T.; Darsalia, V.; Ekdahl, C.T.; Kokaia, Z.; Lindvall, O. Persistent production of neurons from adult brain stem cells during recovery after stroke. Stem Cells 2006, 24, 739-747. [CrossRef] [PubMed]

32. Arvidsson, A.; Collin, T.; Kirik, D.; Kokaia, Z.; Lindvall, O. Neuronal replacement from endogenous precursors in the adult brain after stroke. Nat. Med. 2002, 8, 963-970. [CrossRef]

33. Bendel, O.; Bueters, T.; von Euler, M.; Ogren, S.O.; Sandin, J.; von Euler, G. Reappearance of hippocampal CA1 neurons after ischemia is associated with recovery of learning and memory. J. Cerebr. Blood Flow Met. 2005, 25, 1586-1595. [CrossRef] [PubMed]

34. Bartsch, T.; Wulff, P. The hippocampus in aging and disease: From plasticity to vulnerability. Neuroscience 2015, 309, 1-16. [CrossRef] [PubMed]

35. Han, C.L.; Li, J.Y.; Wang, C.X.; Luo, L.X. Wnt5a through noncanonical Wnt/JNK signaling promotes the differentiation of human embryonic stem cells to lentoid bodies. Investig. Ophth. Vis. Sci. 2018, 59, 891.

36. Doudney, K.; Stanier, P. Epithelial cell polarity genes are required for neural tube closure. Am. J. Med. Genet. C Semin. Med. Genet. 2005, 135, 42-47. [CrossRef]

37. Nishimura, T.; Honda, H.; Takeichi, M. Planar cell polarity links axes of spatial dynamics in neural-tube closure. Cell 2012, 149, 1084-1097. [CrossRef]

38. Zhang, G.L.; Wang, L.H.; Liu, X.Y.; Zhang, Y.X.; Hu, M.Y.; Liu, L.; Fang, Y.Y.; Mu, Y.; Zhao, Y.; Huang, S.H.; et al. Cerebral Dopamine Neurotrophic Factor (CDNF) Has Neuroprotective Effects against Cerebral Ischemia That May Occur through the Endoplasmic Reticulum Stress Pathway. Int. J. Mol. Sci. 2018, 19, 1905. [CrossRef]

(C) 2020 by the authors. Licensee MDPI, Basel, Switzerland. This article is an open access article distributed under the terms and conditions of the Creative Commons Attribution (CC BY) license (http://creativecommons.org/licenses/by/4.0/). 